\title{
Penetration of a wetting front in a porous medium interacting with the flow *
}

\author{
Elena COMPARINI, Paola MANNUCCI \\ Dipartimento di Matematica "U. Dini" \\ Università di Firenze, Viale Morgagni 67/a, Firenze, Italia
}

\begin{abstract}
We analyse a model for incompressible flows through a porous medium, characterized by a mechanical interaction between the flow and the solid matrix and by the presence of a wetting front penetrating in a dry medium.
\end{abstract}

\section{Introduction}

We study a one-dimensional filtration problem describing the flow of an incompressible fluid injected in a dry solid, at a given pressure.

The model considers a porous medium occupying the region $x>0$, where $x$ is the downward directed vertical coordinate. Capillarity is neglected. As a consequence, the penetrating fluid creates a wetting front, denoted by $x=s(t)$, which separates the dry soil from the saturated medium.

In the wet zone the flow causes a deformation of the porous matrix, thus we will assume that the porosity $\epsilon$, which is a prescribed constant in the dry region, is an unknown function of $x$ and $t$.

The hydraulic conductivity $k$ is taken as a function of $\epsilon$ and therefore is also affected.

We neglect macroscopic deformations of the medium and we assume that no chemical process takes place.

The equations governing the flux and the porosity in the saturated region $0<x<s(t)$, are

$$
q=-k(\epsilon) \frac{\partial}{\partial x}(p-\rho g x)
$$

\footnotetext{
*Work partially supported by MURST National Project "Problemi non lineari...".
} 


$$
\frac{\partial \epsilon}{\partial t}=-G(q, \epsilon) \xi\left[\epsilon-\epsilon^{*}(q)\right] .
$$

Equation (1.1) is the Darcy's law: $q$ is the volumetric velocity of the fluid, $p$ is the pressure (prescribed as a given function of $t$ at $x=0$, and zero at the wetting front), $\rho$ is the constant fluid density and $g$ is the gravity acceleration.

Equation (1.2) expresses the decrease of porosity due to the flow-induced compression. $G$ is a positive function, $\xi$ is nonnegative and vanishes when its argument is non-positive. $\epsilon^{*}(q)$ is the lower limit porosity depending on the local values of $q$.

In (1.2) we have assumed that the deformation process, due to the flowinduced compression is irreversible.

This model can be generalized, with minor changes, considering in equation (1.2) an adding term describing the mass release from the porous matrix (see [3]).

A further relationship between flux and porosity is obtained from

$$
\frac{\partial \epsilon}{\partial t}+\frac{\partial q}{\partial x}=0,
$$

expressing mass conservation and saturation.

Since the wetting front moves with the same velocity as the fluid particles, we have

$$
\dot{s}(t)=\frac{1}{\epsilon_{0}} q(s(t), t) .
$$

The constant $\epsilon_{0}$ represents the value of the porosity at $x=s(t)$, which is assumed equal to the value in the dry medium.

The model is then completed by initial and boundary conditions

$$
\begin{aligned}
& \epsilon(s(t), t)=\epsilon_{0}, \\
& p(0, t)=p_{0}(t)>0, \\
& p(s(t), t)=0, \\
& s(0)=0 .
\end{aligned}
$$

We assume that the function $p_{0}(t)$ is continuous and

$$
\pi_{1} t^{n} \leq p_{0}(t) \leq \pi_{2} t^{n},
$$

in a bounded interval, for two positive constants $\pi_{1}, \pi_{2}$ and some real number $n \geq 0$.

We refer to [1], [6] for a review of the literature about non-standard filtration problems of this kind.

In [3] the phenomenon of the flow-induced deformation of the porous matrix, coupled with the mass losses, is considered in the case in which the fluid penetrates through a layer of prescribed thickness, that is in the absence of the free boundary.

Concerning models presenting a wetting front and generalizing the classical Green-Ampt problem ([5]), we quote [2], [4]. 
In [2] the porosity is taken constant, but the dissolution of a substance takes place in the wet region, influencing the hydraulic conductivity.

In [4] the physical parameters of the system $k$ and $\epsilon$ depend on $q$ in a prescribed way.

The aim of this paper is to obtain the global existence and uniqueness of a classical solution for the problem described above.

According to the behaviour of the pressure at $x=0$, we distinguish two cases (see (1.9)): $0 \leq n<1$, in which the initial stage of the problem is driven by the pressure gradient, and $n \geq 1$, corresponding to a dominant influence of gravity.

\section{Statement of the problem}

The governing equations in dimensionless form are the following (Problem I)

$$
\begin{array}{ll}
\frac{\partial \epsilon(x, t)}{\partial t}=-G(q, \epsilon) \xi\left(\epsilon-\epsilon_{*}(q)\right), & 0<x<s(t), t>0, \\
\frac{\partial \epsilon}{\partial t}+\frac{\partial q}{\partial x}=0, & 0<x<s(t), t>0, \\
q(x, t)=-k(\epsilon) \frac{\partial}{\partial x}(p-\rho g x), & 0<x<s(t), t>0, \\
\dot{s}(t)=\frac{1}{\epsilon_{0}} q(s(t), t), & t>0,
\end{array}
$$

with initial and boundary conditions

$$
\begin{array}{cc}
\epsilon(s(t), t)=\epsilon_{0}, & t>0, \\
p(0, t)=p_{0}(t), & t>0, \\
p(s(t), t)=0, & t>0, \\
s(0)=0 . &
\end{array}
$$

We take the following assumptions on the data

$$
\begin{aligned}
& -G\left(\eta_{1}, \eta_{2}\right) \in C^{1}, G \geq 0, \frac{\partial G}{\partial \eta_{1}}, \frac{\partial G}{\partial \eta_{2}} \text { positive bounded; } \\
& -\epsilon_{*}(\eta) \in C^{1}, \quad \frac{d \epsilon_{*}}{d \eta}<0,
\end{aligned}
$$

there exist two constants $\epsilon_{m}, \epsilon_{M}$ such that

$0<\epsilon_{m} \leq \epsilon_{*}(\eta) \leq \epsilon_{M}<1, \forall \eta, \epsilon_{m} \leq \epsilon_{0} \leq \epsilon_{M} ;$

$-\xi(\eta) \in C^{1}, 0 \leq \xi \leq \xi_{0}, \xi(\eta)=0$ for $\eta<0$,

$0 \leq \xi^{\prime} \leq \xi_{0}^{\prime}$, for some positive constants $\xi_{0}, \xi_{0}^{\prime}$, and $|\eta|<\epsilon_{M}$;

$-k(\eta) \in C^{1}, 0<k_{m} \leq k(\eta) \leq k_{M}$

$\left|k^{\prime}\right| \leq k_{0}^{\prime}, \forall \eta$, for some positive constants $k_{m}, k_{M}, k_{0}^{\prime}$;

- $p_{0}(t) \in C^{1}(0, T), \pi_{1} t^{n} \leq p_{0}(t) \leq \pi_{2} t^{n}, \pi_{1}, \pi_{2}$ constants and $n \geq 0$ in some bounded interval $[0, T]$. 
Definition 2.1 A solution of Problem I is $(T, s, p, \epsilon, q)$ such that

1) $T>0$,

2) $s(t) \in C^{1}(0, T) \cap C([0, T])$,

$p(x, t) \in C^{1,0}\left(Q_{T}\right)$,

$\epsilon(x, t) \in C^{1,1}\left(Q_{T}\right)$,

$q(x, t) \in C^{1,0}\left(Q_{T}\right)$,

where $Q_{T}=\{(x, t): 0<x<s(t), 0<t<T\}$.

3) The functions $s, p, \epsilon, q$ satisfy equations (2.1)-(2.4) in $Q_{T}$ with (2.5)-(2.8).

Theorem 2.1 Suppose that assumptions (2.9)-(2.13) hold. Then

a) If $0 \leq n<1$, Problem I admits one unique solution for any $T>0$.

b) If $n \geq 1$, Problem I admits at least one solution for any $T>0$.

The proof of this theorem is based upon a fixed point argument and the procedure goes through the following steps:

i) We introduce the set

$$
\begin{aligned}
\Sigma=\left\{\sigma \in C^{1}(0, T):\right. & \sigma(0)=0 ; A_{1}(t) \leq \sigma(t) \leq A_{2}(t) ; \\
& \left.B_{1}(t) \leq \dot{\sigma}(t) \leq B_{2}(t) ; \frac{\left|\dot{\sigma}\left(t_{1}\right)-\dot{\sigma}\left(t_{2}\right)\right|}{\left|t_{1}-t_{2}\right|} \leq S\left(t_{1}, t_{2}\right)\right\},
\end{aligned}
$$

where $A_{i}(t), B_{i}(t), i=1,2, S\left(t_{1}, t_{2}\right)$ and $T$ will be defined in the following sections. ii) We take any $\sigma \in \Sigma$ and we define $s(t)=\sqrt{\sigma(t)}$. In order to obtain $q(x, t)$ and $\epsilon(x, t)$ corresponding to $s(t)=\sqrt{\sigma(t)}$, we consider the following ODE's system with the appropriate initial conditions,

$$
\begin{array}{cc}
q_{x}=\Gamma(q, \epsilon), & \text { in } Q_{T}, \\
\epsilon_{t}=-\Gamma(q, \epsilon), & \text { in } Q_{T}, \\
q(s(t), t)=\epsilon_{0} \dot{s}, & 0<t<T, \\
\epsilon(s(t), t)=\epsilon_{0}, & 0<t<T,
\end{array}
$$

where we set

$$
\Gamma(q, \epsilon)=G(q, \epsilon) \xi\left(\epsilon-\epsilon_{*}(q)\right) .
$$

System (2.14) cannot be reduced to a vectorial ODE for $(q, \epsilon)$.

A proof of existence and uniqueness of the solution of (2.14) will be obtained by means of a fixed point argument. 
iii) We obtain $p(x, t)$ by integrating

$$
\begin{aligned}
& p_{x}=-\frac{q}{k(\epsilon)}+\rho g, \\
& p(0, t)=p_{0}(t) .
\end{aligned}
$$

iv) The next step consists in determining a function $\widetilde{\sigma}(t)$ associated to $\sigma(t)$, defined by the operator $\mathcal{T}: \Sigma \rightarrow \Sigma$, such that $\mathcal{T} \sigma=\widetilde{\sigma}$.

The operator $\mathcal{T}$ can be introduced in the following way (see [2]): from (2.15), (2.7) we obtain

$$
0=p_{0}(t)-\int_{0}^{\sqrt{\sigma(t)}} \frac{q(y, t)}{k(\epsilon(y, t))} d y+\rho g \sqrt{\sigma(t)} .
$$

From (2.14) we have

$$
q(x, t)=q(\sqrt{\sigma(t)}, t)-\int_{\sqrt{\sigma(t)}}^{x} \Gamma(q, \epsilon)(y, t) d y
$$

Inserting (2.17) in (2.16), we obtain an expression for $\dot{\sigma}=2 s \dot{s}$ that can be integrated with the initial condition $\sigma(0)=0$. Hence the operator $\mathcal{T}$ is defined by

$$
\begin{gathered}
\tilde{\sigma}(t)=2 \int_{0}^{t}\left[p_{0}(\tau)+\rho g \sqrt{\sigma(\tau)}+\int_{0}^{\sqrt{\sigma(\tau)}} R(\epsilon(x, \tau)) \times\right. \\
\left.\int_{x}^{\sqrt{\sigma(\tau)}} \Gamma(q(y, \tau), \epsilon(y, \tau)) d y d x\right] d \tau / \frac{\epsilon_{0}}{\sqrt{\sigma(t)}} \int_{0}^{\sqrt{\sigma(\tau)}} R(\epsilon(x, \theta)) d \theta
\end{gathered}
$$

where

$$
R(\epsilon)=\frac{1}{k(\epsilon)} .
$$

Any fixed point $\sigma=\widetilde{\sigma}$ of (2.18) with the corresponding $s=\sqrt{\sigma}, q, \epsilon, p$, obtained by $(2.14),(2.15)$ gives a solution of Problem I.

We will show that once a fixed point has been found, global existence can be proved extending the solution for any $T>0$.

Next sections will be devoted to the proof of Theorem 2.1, in the case $0 \leq$ $n<1$ and $n \geq 1$ respectively. 


\section{Case $0 \leq n<1$}

Take $A_{i}(t), B_{i}(t), i=1,2$ in the definition of $\Sigma$ in the following form:

$$
\begin{aligned}
& A_{1}(t)=\frac{2 k_{m} \pi_{1}}{\epsilon_{0}(n+1)} t^{n+1} \equiv \alpha_{1} t^{n+1}, \\
& A_{2}(t)=\alpha_{2} t^{n+1},
\end{aligned}
$$

where $\alpha_{2}$ is the unique positive root of

$$
\begin{aligned}
& \alpha_{2}=\frac{2 k_{M}}{\epsilon_{0}}\left[\frac{\pi_{2}}{n+1}+2 \rho g \frac{\sqrt{\alpha_{2}}}{n+3} T^{(1-n) / 2}+\frac{\Gamma_{0} \alpha_{2} T}{2 k_{m}(n+2)}\right], \\
& B_{1}(t)=\frac{2 k_{m} \pi_{1}}{\epsilon_{0}} t^{n} \equiv \beta_{1} t^{n}, \\
& B_{2}(t)=\frac{2 k_{M}}{\epsilon_{0}}\left(\pi_{2}+\rho g \sqrt{\alpha_{2}} T^{(1-n) / 2}+\frac{\Gamma_{0} \alpha_{2} T}{2 k_{m}}\right) t^{n} \equiv \beta_{2} t^{n},
\end{aligned}
$$

for any $t \in(0, T)$, with $T$ fixed. $S\left(t_{1}, t_{2}\right)$ will be specified later.

Taken any $\sigma \in \Sigma$, we calculate $s=\sqrt{\sigma}$ and solve system (2.14) using a fixed point argument.

Lemma 3.1 Under assumptions (2.9)-(2.10), problem (2.14) has a unique solution $(q, \epsilon)$ where $q(x, t) \in C^{1,0}\left(Q_{T}\right), \epsilon(x, t) \in C^{1,1}\left(Q_{T}\right)$ for any prescribed $\sigma \in \Sigma$.

Proof. We define the set

$$
\begin{aligned}
\Theta=\{ & q(x, t) \in C^{0}\left(Q_{T}\right), q(s(t), t)=\epsilon_{0} \dot{s}(t), \\
& \left.0<q_{m}(t) \leq q(x, t) \leq q_{M}(t), 0<t<T\right\}
\end{aligned}
$$

with $q_{m}, q_{M}, T$ to be determined later, in dependence on $A_{i}, B_{i}$.

For any $q \in \Theta$ we compute

$$
\epsilon(x, t)=\epsilon_{0}-\int_{s^{-1}(x)}^{t} \Gamma(q, \epsilon)(x, \tau) d \tau,
$$

then we define the operator

$$
\Phi: \Theta \longrightarrow C^{0}, \quad \Phi q=\widetilde{q},
$$

where

$$
\widetilde{q}(x, t)=\epsilon_{0} \dot{s}(t)-\int_{x}^{s(t)} \Gamma(q, \epsilon)(y, t) d y .
$$


First let us prove some a priori estimates. We have

$$
\begin{aligned}
& \sqrt{A_{1}(t)} \leq s(t) \leq \sqrt{A_{2}(t)}, \quad t \geq 0 \\
& \frac{B_{1}(t)}{2 \sqrt{A_{2}(t)}} \leq \dot{s}(t) \leq \frac{B_{2}(t)}{2 \sqrt{A_{1}(t)}}, \quad t>0
\end{aligned}
$$

Recalling (3.1)-(3.5), the above estimates can be expressed in the form

$$
\begin{aligned}
& \sqrt{\alpha_{1}} t^{(n+1) / 2} \leq s(t) \leq \sqrt{\alpha_{2}} t^{(n+1) / 2}, \quad t \geq 0 \\
& \frac{\beta_{1}}{2 \sqrt{\alpha_{2}}} t^{(n-1) / 2} \leq \dot{s}(t) \leq \frac{\beta_{2}}{2 \sqrt{\alpha_{1}}} t^{(n-1) / 2}, \quad t>0 .
\end{aligned}
$$

Note that, because of assumptions (2.9)-(2.10), and recalling that $\epsilon_{t}<0$ we have

$$
\epsilon_{m} \leq \epsilon(x, t) \leq \epsilon_{0}, \quad \forall(x, t) \in Q_{T},
$$

where $\epsilon_{m}$ is defined by (2.10).

From (3.7), we get

$$
q_{m}(t) \leq \widetilde{q}(x, t) \leq q_{M}(t)
$$

where

$$
\begin{aligned}
q_{m}(t) & =\frac{\epsilon_{0} \beta_{1}}{2 \sqrt{\alpha_{2}}} t^{(n-1) / 2}-\Gamma_{0} \sqrt{\alpha_{2}} t^{(n+1) / 2}, \\
q_{M}(t) & =\frac{\epsilon_{0} \beta_{2}}{2 \sqrt{\alpha_{1}}} t^{(n-1) / 2}
\end{aligned}
$$

with $\Gamma_{0}=\sup _{\substack{q \in \Theta \\ \epsilon \in(0,1)}}|\Gamma(q, \epsilon)|$.

Note that $q_{m}(t)>0$ for

$$
t<\frac{\epsilon_{0} \beta_{1}}{2 \alpha_{2} \Gamma_{0}}
$$

Hence $\Phi(\Theta) \subset \Theta$.

Now we show that $\Phi$ is contractive. Let $q_{1}, q_{2} \in \Theta$ and $\epsilon_{1}, \epsilon_{2}$ be the corresponding functions defined by (3.6). Denoted by $E(x, t)$ the difference $\epsilon_{1}(x, t)-$ $\epsilon_{2}(x, t), E$ satisfies

$$
\begin{aligned}
& E_{t}=\left.\Gamma_{\epsilon}\right|_{\epsilon=\bar{\epsilon}} E+\left.\Gamma_{q}\right|_{q=\bar{q}}\left(q_{1}-q_{2}\right), \\
& E(s(t), t)=0,
\end{aligned}
$$

with $\bar{\epsilon}, \bar{q}$ suitable functions.

Hence

$$
\left\|\epsilon_{1}-\epsilon_{2}\right\|_{T} \leq C_{1}(T)\left\|q_{1}-q_{2}\right\|_{T},
$$

where, recalling assumptions on $\Gamma_{\epsilon}$ and $\Gamma_{q}, C_{1}(t)$ is a nondecreasing function of $t$, and

$$
\left\|\widetilde{q}_{1}-\widetilde{q}_{2}\right\|_{T} \leq C_{2}(T)\left\|q_{1}-q_{2}\right\|_{T},
$$


where

$$
C_{2}(t)=\left(\bar{\Gamma}_{q}+C_{1}(T)\right) \sqrt{A_{2}(t)} .
$$

Here and in the following we will define:

$$
\|f\|_{T}=\sup _{\substack{0<x<s(t) \\ 0<t<T}}|f(x, t)|, \quad \bar{\Gamma}_{q}=\left\|\frac{\partial \Gamma}{\partial q}\right\|_{T}, \quad \bar{\Gamma}_{\epsilon}=\left\|\frac{\partial \Gamma}{\partial \epsilon}\right\|_{T}
$$

while $C_{i}(t), c_{i}(t)$ will denote suitable sufficiently regular nondecreasing functions which can be expressed in terms of the data.

Recalling (3.2) we have that $C_{2}(t)<1$ for

$$
t<\left(\frac{1}{\sqrt{\alpha_{2}}\left(\bar{\Gamma}_{q}+C_{1}(T)\right)}\right)^{2 /(n+1)} .
$$

Then $\Phi$ is a contraction map for $T$ sufficiently small.

Further regularity on $q(x, t)$ and $\epsilon(x, t)$ can be obtained in the following way: from (2.14) we have

$$
\begin{aligned}
& 0 \leq q_{x}(x, t) \leq \Gamma_{0}, \\
& -\Gamma_{0} \leq \epsilon_{t}(x, t) \leq 0
\end{aligned}
$$

moreover the estimate

$$
\left|\epsilon_{x}(x, t)\right| \leq C_{3}(T)+C_{4}(T) t^{(1-n) / 2},
$$

for any $x \in(0, s(t))$, is obtained noting that $\epsilon_{x}$ satisfies

$$
\begin{aligned}
& \left(\epsilon_{x}\right)_{t}+\Gamma_{\epsilon}\left(\epsilon_{x}\right)+\Gamma \Gamma_{q}=0, \\
& \epsilon_{x}(s(t), t)=\frac{\Gamma(s(t), t)}{\dot{s}(t)} .
\end{aligned}
$$

Set now $Q(x) \equiv q\left(x, t_{1}\right)-q\left(x, t_{2}\right), 0<t_{1}<t_{2}<T, Q(x)$ satisfies the following integral equation

$$
\begin{aligned}
Q(x)= & -\int_{x}^{s\left(t_{1}\right)} \Gamma_{q}(\bar{q}, \epsilon) Q(y) d y+\epsilon_{0}\left(\dot{s}\left(t_{1}\right)-\dot{s}\left(t_{2}\right)\right)- \\
& -\int_{x}^{s\left(t_{1}\right)} \Gamma_{\epsilon}(\bar{q}, \epsilon) \Gamma(y, \bar{t})\left(t_{1}-t_{2}\right) d y+\int_{s\left(t_{1}\right)}^{s\left(t_{2}\right)} \Gamma(q, \epsilon)\left(y, t_{2}\right) d y
\end{aligned}
$$

with suitable $\bar{t} \in\left(t_{1}, t_{2}\right)$, and $\bar{q}$. 
Using Gronwall's Lemma we have

$$
\begin{aligned}
|Q(x)| & \leq\left|t_{1}-t_{2}\right|\left[1+C_{5}(T)\right]\left(\epsilon_{0} S\left(t_{1}, t_{2}\right)+C_{6} t_{2}^{(n+1) / 2}+C_{7} t_{1}^{(n-1) / 2}\right) \equiv \\
& \equiv A\left(t_{1}, t_{2}\right)\left|t_{1}-t_{2}\right| .
\end{aligned}
$$

Note that the function $A\left(t_{1}, t_{2}\right)$ depends on $S\left(t_{1}, t_{2}\right)$ and then it will be determined later. However we can note here that $A\left(t_{1}, t_{2}\right)$ is unbounded when $t_{1} \rightarrow 0$.

The regularity of $p(x, t)$ follows from (2.3), taking into account Lemma 3.1.

Proof of Theorem 2.1-a) Take any $\sigma \in \Sigma$. We have immediately that $\widetilde{\sigma}(t)$ defined in (2.18) satisfies

$$
\alpha_{1} t^{n+1} \leq \widetilde{\sigma}(t) \leq \alpha_{2} t^{n+1},
$$

where $\alpha_{1}, \alpha_{2}$ are defined by (3.1), (3.3) and

$$
\beta_{1} t^{n} \leq \dot{\tilde{\sigma}}(t) \leq \beta_{2} t^{n},
$$

where $\beta_{1}, \beta_{2}$ are defined by (3.4), (3.5). Moreover, noting that

$$
\frac{1}{\sqrt{\sigma}} \int_{0}^{\sqrt{\sigma}} R(\epsilon(x, t)) d x=R(\epsilon(\bar{x}, t)) \equiv \bar{R}(t),
$$

with $\bar{x}$ suitable value in $(0, \sqrt{\sigma})$, we have for $0<t_{1}<t_{2}<T$ :

$$
\begin{aligned}
& \left|\dot{\tilde{\sigma}}\left(t_{1}\right)-\dot{\tilde{\sigma}}\left(t_{2}\right)\right|=\frac{2}{\epsilon_{0}} \mid \frac{p_{0}\left(t_{1}\right)}{\bar{R}\left(t_{1}\right)}-\frac{p_{0}\left(t_{2}\right)}{\bar{R}\left(t_{2}\right)}+\rho g\left(\frac{\sqrt{\sigma\left(t_{1}\right)}}{\bar{R}\left(t_{1}\right)}-\frac{\sqrt{\sigma\left(t_{2}\right)}}{\bar{R}\left(t_{2}\right)}\right)+ \\
& +\frac{1}{\bar{R}\left(t_{1}\right)} \int_{0}^{\sqrt{\sigma\left(t_{1}\right)}} R\left(\epsilon\left(x, t_{1}\right)\right) \int_{x}^{\sqrt{\sigma\left(t_{1}\right)}} \Gamma(q, \epsilon)\left(y, t_{1}\right) d y d x- \\
& -\frac{1}{\bar{R}\left(t_{2}\right)} \int_{0}^{\sqrt{\sigma\left(t_{2}\right)}} R\left(\epsilon\left(x, t_{2}\right)\right) \int_{x}^{\sqrt{\sigma\left(t_{2}\right)}} \Gamma(q, \epsilon)\left(y, t_{2}\right) d y d x \mid .
\end{aligned}
$$

Let us estimate now the terms in the right-hand side of (3.26). We have:

$$
\begin{aligned}
& \left|p_{0}\left(t_{1}\right)-p_{0}\left(t_{2}\right)\right|=\left|\dot{p}_{0}(\bar{t})\right|\left|t_{1}-t_{2}\right|, \quad \bar{t} \in\left[t_{1}, t_{2}\right], \\
& \left|\frac{1}{\bar{R}\left(t_{1}\right)}-\frac{1}{\bar{R}\left(t_{2}\right)}\right| \leq k_{0}^{\prime} \Gamma_{0}\left|t_{1}-t_{2}\right|, \\
& \left|\Gamma(q, \epsilon)\left(x, t_{1}\right)-\Gamma(q, \epsilon)\left(x, t_{2}\right)\right| \leq\left\{\Gamma_{q}(q, \epsilon)(x, \bar{t}) A\left(t_{1}, t_{2}\right)+\right. \\
& \left.+\Gamma \Gamma_{\epsilon}(q, \epsilon)(x, \bar{t})\right\}\left|t_{1}-t_{2}\right|, \\
& \left|\sqrt{\sigma\left(t_{1}\right)}-\sqrt{\sigma\left(t_{2}\right)}\right|=\frac{\dot{\sigma}(\bar{t})}{2 \sqrt{\sigma(\bar{t})}}\left|t_{1}-t_{2}\right| \leq \frac{\beta_{2}}{2 \sqrt{\alpha_{1}}} t_{1}^{(n-1) / 2}\left|t_{1}-t_{2}\right| .
\end{aligned}
$$


Then

$$
\begin{aligned}
& \left|\dot{\widetilde{\sigma}}\left(t_{1}\right)-\dot{\tilde{\sigma}}\left(t_{2}\right)\right| \leq \frac{2}{\epsilon_{0}}\left\{k_{M} \sup _{t \in\left[t_{1}, t_{2}\right]}\left|\dot{p}_{0}(t)\right|+\frac{k_{M} \alpha_{2}}{k_{m} 2} \bar{\Gamma}_{q} A\left(t_{1}, t_{2}\right) t_{2}^{n+1}\right. \\
& \left.+\rho g \frac{k_{M} \beta_{2}}{\sqrt{\alpha_{1}}} t_{1}^{(n-1) / 2}+C_{8}(T)\right\}\left|t_{1}-t_{2}\right| \equiv \\
& \equiv S\left(t_{1}, t_{2}\right)\left|t_{2}-t_{2}\right| .
\end{aligned}
$$

Recalling (3.22), we have

$$
S\left(t_{1}, t_{2}\right)=\frac{\frac{2}{\epsilon_{0}} k_{M} \sup _{t \in\left[t_{1}, t_{2}\right]}\left|\dot{p}_{0}(t)\right|+C_{9}(T) t_{1}^{(n-1) / 2}+C_{10}(T)}{1-\frac{k_{M} \alpha_{2}}{k_{m}} \bar{\Gamma}_{q} T^{n+1}\left(1+C_{5}(T)\right)},
$$

for $T$ sufficiently small.

Note that (3.28) gives an estimate for the Lipschitz constant of $\dot{\widetilde{\sigma}}(t)$ away from $t=0$. In fact both terms $\dot{p}_{0}(t)$ and $t^{(n-1) / 2}$ are unbounded for $t=0$.

The definition of $A\left(t_{1}, t_{2}\right)$ (see (3.22) in Lemma 3.1) follows immediately from (3.28). Note that the behaviour of $A\left(t_{1}, t_{2}\right)$ is the same as the behaviour of $S\left(t_{1}, t_{2}\right)$.

Hence from $(3.23),(3.24),(3.27)$ and $(3.28) \mathcal{T}(\Sigma)=\Sigma$.

Next we show that $\mathcal{T}$ is a contractive operator in the $C^{1}$-norm.

Given $\sigma_{1}, \sigma_{2} \in \Sigma$, and the corresponding $q_{1}, q_{2}, \epsilon_{1}, \epsilon_{2}$, recalling the definition of $\mathcal{T}$,

$$
\begin{aligned}
& \frac{\epsilon_{0}}{2}\left|\dot{\tilde{\sigma}}_{1}-\dot{\sigma}_{2}\right| \leq\left[\pi_{2} t^{n}+\rho g \sqrt{\alpha_{2}} t^{(n+1) / 2}+\frac{k_{M} \Gamma_{0} \alpha_{2}}{2} t^{n+1}+\right. \\
& \left.+\frac{k_{M} \Gamma_{0} \alpha_{2}}{2 k_{m}^{2}} t^{n+1}\right] k_{0}^{\prime}\left|\epsilon_{1}-\epsilon_{2}\right|+\frac{\alpha_{2} k_{M}}{2 k_{m}} t^{n+1}\left|\Gamma\left(q_{1}, \epsilon_{1}\right)-\Gamma\left(q_{2}, \epsilon_{2}\right)\right|+ \\
& +\left[\frac{k_{M}}{k_{m}} \Gamma_{0}\left(\sqrt{\alpha_{2}} t^{(n+1) / 2}+\frac{1}{2}\left|\sqrt{\sigma_{1}}-\sqrt{\sigma_{2}}\right|\right)+\rho g k_{M}\right]\left|\sqrt{\sigma_{1}}-\sqrt{\sigma_{2}}\right| .
\end{aligned}
$$

Noting that

$$
\begin{aligned}
& \left|\Gamma\left(q_{1}, \epsilon_{1}\right)-\Gamma\left(q_{2}, \epsilon_{2}\right)\right| \leq \bar{\Gamma}_{q}\left|q_{1}-q_{2}\right|+\bar{\Gamma}_{\epsilon}\left|\epsilon_{1}-\epsilon_{2}\right| \\
& \left|\epsilon_{1}-\epsilon_{2}\right| \leq\left|\Gamma\left(q_{1}, \epsilon_{1}\right)-\Gamma\left(q_{2}, \epsilon_{2}\right)\right| t+\frac{\Gamma_{0}}{b_{1}(t)}\left|\sqrt{\sigma_{1}}-\sqrt{\sigma_{2}}\right|, \\
& \left|q_{1}-q_{2}\right| \leq \frac{\epsilon_{0}}{2}\left|\frac{\dot{\sigma}_{1}}{\sqrt{\sigma_{1}}}-\frac{\dot{\sigma}_{2}}{\sqrt{\sigma_{2}}}\right|+a_{2}(t)\left|\Gamma\left(q_{1}, \epsilon_{1}\right)-\Gamma\left(q_{2}, \epsilon_{2}\right)\right|+ \\
& +\Gamma_{0}\left|\sqrt{\sigma_{1}}-\sqrt{\sigma_{2}}\right|, \\
& \left|\sqrt{\sigma_{1}}-\sqrt{\sigma_{2}}\right| \leq \frac{1}{2 \sqrt{\alpha_{1}} t(n+1) / 2}\left|\sigma_{1}-\sigma_{2}\right|
\end{aligned}
$$


from which:

$$
\begin{aligned}
& \left\|q_{1}-q_{2}\right\|_{t} \leq \frac{1}{1-\Gamma_{\epsilon} T-\sqrt{A_{2}(T)} \bar{\Gamma}_{q}}\left[\frac{\epsilon_{0}}{2 \sqrt{\alpha_{1}}} t^{-(n+1) / 2}\left\|\dot{\sigma}_{1}-\dot{\sigma}_{2}\right\|_{t}+\right. \\
& +\left(\frac{\Gamma_{0} \bar{\Gamma}_{\epsilon}}{1-\bar{\Gamma}_{\epsilon} T} \frac{\alpha_{2}}{2 \sqrt{\alpha_{1}} \beta_{1}} t^{(1-n) / 2}+\right. \\
& \left.\left.+\frac{1}{2 \sqrt{\alpha_{1}}}\left(\Gamma_{0}+\frac{\epsilon_{0} \sqrt{\alpha_{2}}}{2 \sqrt{\alpha_{1}}}\right) t^{-(n+1) / 2}\right)\left\|\sigma_{1}-\sigma_{2}\right\|_{t}\right], \\
& \left\|\epsilon_{1}-\epsilon_{2}\right\|_{t} \leq \frac{\bar{\Gamma}_{q}}{1-\Gamma_{\epsilon} T-\sqrt{A_{2}(T)} \bar{\Gamma}_{q}}\left[\frac{\epsilon_{0}}{2 \sqrt{\alpha_{1}}} t^{(1-n) / 2}\left\|\dot{\sigma}_{1}-\dot{\sigma}_{2}\right\|_{t}+\right. \\
& +\left(\frac{\Gamma_{0} \bar{\Gamma}_{\epsilon}}{1-\bar{\Gamma}_{\epsilon} T} \frac{\alpha_{2}}{2 \sqrt{\alpha_{1}} \beta_{1}} t^{(3-n) / 2}+\left(\frac{\Gamma_{0}}{2 \sqrt{\alpha_{1}}}+\frac{\epsilon_{0} \sqrt{\alpha_{2}}}{4 \alpha_{1}}+\right.\right. \\
& \left.\left.\left.+\frac{1}{1-\bar{\Gamma}_{\epsilon} T} \frac{\Gamma_{0}}{\Gamma_{q}} \frac{\sqrt{\alpha_{2}}}{\sqrt{\alpha_{1}} \beta_{1}}\right) t^{(1-n) / 2}\right)\left\|\sigma_{1}-\sigma_{2}\right\|_{t}\right]
\end{aligned}
$$

with $T$ such that

$$
1-\Gamma_{\epsilon} T-\sqrt{A_{2}(T)} \bar{\Gamma}_{q}>0 .
$$

Finally, from (3.30), (3.31), then (3.29) reduces to

$$
\begin{aligned}
& \left\|\dot{\tilde{\sigma}}_{1}-\dot{\tilde{\sigma}}_{2}\right\|_{t} \leq C_{11}(T)\left(t^{(n+3) / 2}+t+t^{(n+1) / 2}\right)\left\|\dot{\sigma}_{1}-\dot{\sigma}_{2}\right\|_{t}+ \\
& +C_{12}(T)\left(t^{(n+5) / 2}+t^{2}+t^{(n+3) / 2}+t+t^{(n+1) / 2}+1\right)\left\|\sigma_{1}-\sigma_{2}\right\|_{t}+ \\
& C_{13} t^{-(n+1) / 2}\left\|\sigma_{1}-\sigma_{2}\right\|_{t} \leq C(T) t^{(1-n) / 2}\left\|\dot{\sigma}_{1}-\dot{\sigma}_{2}\right\|_{t} .
\end{aligned}
$$

The constants $C_{11}, C_{12}, C_{13}$ depend on the data and on $T$.

(3.33) shows that $\mathcal{T}$ is continuous in the $C^{1}$-norm for any $T$ and in particular, recalling that $0 \leq n<1$, is contractive for $T$ small enough, such that

$$
C(T) T^{(1-n) / 2}<1 .
$$

Note that the maximum existence time $T$ is defined recalling also (3.16), (3.20), (3.28), (3.32).

Global existence follows extending the solution after $T$.

We can consider a problem like Problem I in $Q_{\bar{T}}=\left\{(x, t): 0<x<s(t), T_{0}<t<\right.$ $\bar{T}\}, T_{0}<T$, prescribing initial data at $t=T_{0}$ obtained from the previous solution.

Then $\epsilon(x, t)$ solves

$$
\epsilon(x, t)= \begin{cases}\epsilon_{0}-\int_{s^{-1}(x)}^{t} \Gamma(q, \epsilon)(x, \tau) d \tau, & x \in\left[s\left(T_{0}\right), s(t)\right], \\ \epsilon\left(x, T_{0}\right)-\int_{T_{0}}^{t} \Gamma(q, \epsilon)(x, \tau) d \tau, & x \in\left[0, s\left(T_{0}\right)\right],\end{cases}
$$


and $q(x, t), p(x, t)$ can be expressed by

$$
\begin{aligned}
& q(x, t)=\epsilon_{0} \dot{s}(t)-\int_{x}^{s(t)} \Gamma(q, \epsilon) d y, \\
& p(x, t)=p_{0}(t)-\int_{0}^{x} \frac{q(y, t)}{k(\epsilon(y, t))} d y+\rho g x .
\end{aligned}
$$

Noting that estimates proved in Lemma 3.1 and Theorem 2.1 hold also in $Q_{\bar{T}}$, we get the result.

Remark 3.1 Note that the assumption $0 \leq n<1$ implies that $\dot{p}_{0}(0)$ is singular, and consequently $\dot{s}$ and $q$ have the same behaviour at $t=0$.

This corresponds to the physical assumption of high pressure gradient.

\section{Case $n \geq 1$}

In this case the functions $A_{i}(t), B_{i}(t), i=1,2$ in the definition of $\Sigma$ are

$$
\begin{aligned}
& A_{1}(t)=\left(\frac{2 k_{m}}{\epsilon_{0}} \rho g\right)^{2} t^{2} \equiv \alpha_{1} t^{2}, \\
& A_{2}(t)=\alpha_{2} t^{2}
\end{aligned}
$$

where $\alpha_{2}$ is the unique positive root of

$$
\begin{aligned}
& \frac{2 k_{m}}{\epsilon_{0}}\left[\frac{\pi_{2}}{n+1} T^{n-1}+\frac{\rho g \sqrt{\alpha_{2}}}{2}+\frac{\Gamma_{0} \alpha_{2}}{6 k_{m}} T\right]=\alpha_{2}, \\
& B_{1}(t)=\left(\frac{2 k_{m}}{\epsilon_{0}} \rho g\right)^{2} t \equiv \beta_{1} t, \\
& B_{2}(t)=\frac{2 k_{M}}{\epsilon_{0}}\left(\pi_{2} T^{n-1}+\frac{\alpha_{2} \Gamma_{0} T}{2 k_{m}}+\rho g \sqrt{\alpha_{2}}\right) t \equiv \beta_{2} t,
\end{aligned}
$$

for any $t \in(0, T)$, with $T$ fixed, $S\left(t_{1}, t_{2}\right)$ will be specified later.

Lemma 3.1 still holds in the case $n \geq 1$ taking into account that

$$
\begin{aligned}
& \sqrt{\alpha_{1}} t \leq s(t) \leq \sqrt{\alpha_{2}} t, \quad t \geq 0, \\
& \frac{\beta_{1}}{2 \sqrt{\alpha_{2}}} \leq \dot{s}(t) \leq \frac{\beta_{2}}{2 \sqrt{\alpha_{1}}}, \quad t \geq 0,
\end{aligned}
$$

where $\alpha_{i}, \beta_{i}, i=1,2$, are defined by (4.1)-(4.5). 
The estimates on the solution in this case are the following

$$
\begin{aligned}
& q_{m}(t) \equiv \frac{\epsilon_{0} \beta_{1}}{2 \sqrt{\alpha_{2}}}-\Gamma_{0} \sqrt{\alpha_{2}} t \leq q(x, t) \leq \frac{\epsilon_{0} \beta_{2}}{2 \sqrt{\alpha_{1}}} \equiv q_{M}, \\
& 0 \leq q_{x}(x, t) \leq \Gamma_{0}, \\
& -\Gamma_{0} \leq \epsilon_{t}(x, t) \leq 0, \quad \text { in } \bar{Q}_{T}, \\
& \left|\epsilon_{x}(x, t)\right| \leq c_{1}(T), \quad \\
& \left|q\left(x, t_{1}\right)-q\left(x, t_{2}\right)\right| \leq\left(1+c_{2}(T)\right)\left(\epsilon_{0} S\left(t_{1}, t_{2}\right)+c_{3}(T)\right)\left|t_{1}-t_{2}\right|,
\end{aligned}
$$

with $S\left(t_{1}, t_{2}\right)$ to be determined.

\section{Proof of Theorem 2.1-b}

The proof of $\mathcal{T}(\Sigma) \subset \Sigma$ follows the same steps as in previous case taking into account (4.1)-(4.5). Moreover

$$
S\left(t_{1}, t_{2}\right)=\frac{\left(2 / \epsilon_{0}\right) k_{M} \sup _{t \in\left[t_{1}, t_{2}\right]}\left|\dot{p}_{0}(t)\right|+c_{4}(T)}{1-\left(k_{M} / k_{m}\right) \alpha_{2} \bar{\Gamma}_{q}\left(1+c_{2}(T)\right) T^{2}} .
$$

Hence, in this case, from (2.13), $S\left(t_{1}, t_{2}\right)$ remains bounded in $t_{1}=0$.

As far as the continuity of operator $\mathcal{T}$ is concerned, we may use the same techniques as in the case $0 \leq n<1$ and taking into account of (3.33) and noting that $C_{13}$ is a constant,

$$
\left\|\dot{\tilde{\sigma}}_{1}-\dot{\widetilde{\sigma}}_{2}\right\|_{t} \leq\left(c(T) t+C_{13}\right)\left\|\dot{\sigma}_{1}-\dot{\sigma}_{2}\right\|_{t} .
$$

Expression (4.14) shows that the operator $\mathcal{T}$ is continuous on $\Sigma$ but in general not contractive.

Remark 4.1 The boundedness of $\dot{p}_{0}(0)$ yields $q(x, t) \in C^{1, \alpha}\left(\bar{Q}_{T}\right)$ and $s(t) \in$ $C^{1+\alpha}([0, T])$. Note that now we have to introduce into the definition of the space $\Sigma$ the condition $\dot{\sigma}(0)=0$.

In this case, $\dot{s}(0)$ can be obtained by differentiating (2.16):

$$
\dot{p}_{0}(0)=\dot{s}(0)\left[\frac{\epsilon_{0} \dot{s}(0)}{k\left(\epsilon_{0}\right)}-\rho g\right] .
$$

Remark 4.2 In the case $n \geq 1$, non-uniqueness is a consequence of non-Lipschitz continuity with respect to $\sigma$ of the right hand-side of (2.18), due to the term $\rho g \sqrt{\sigma}$.

Uniqueness can be proved if gravity is neglected. In fact, taking into account (4.14) and recalling that $C_{13}=\frac{\rho g k_{M}}{\epsilon_{0} \sqrt{\alpha_{1}}}$, we note that the operator $\mathcal{T}$ is contractive for $T$ sufficiently small. 


\section{References}

[1] A. FASANO, Some non-standard one-dimensional filtration problems, Lecture at Chiba University, to appear

[2] A. FASANO. The penetration of a wetting front through a porous medium accompanied by the dissolution of a substance, Int. Conf. Flow through Porous media, C. Carasso et al. eds. (1995)

[3] A. FASANO, M. PRIMICERIO, Flows through saturated mass exchanging porous media under high pressure gradients, in Calculus of variations, applications and computation, Pont a Mousson, C. Bandle et al. eds., Pitmann Press Notes Math. 326, 109-129 (1994).

[4] A. FASANO, P. TANI, Penetration of a wetting front in a porous medium with flux dependent hydraulic parameters, Nonlinear Problems in Applied Mathematics (1995), to appear.

[5] W.H. GREEN, G.A. AMPT. Studies on soil physics. The flow of air and water through soils, J. Agric. Sci. 4, 1-24 (1911).

[6] L. PREZIOSI, D.D. JOSEPH, G. BEAVERS, Infiltration of initially dry, deformable porous media, to appear in J. of Multiphase Flows.

Received February 19, 1996 